\title{
Focus group
}

David F Marks

Potential competing interests: The author(s) declared that no potential competing interests exist.

Focus groups comprise one or more group discussions in which participants 'focus' collectively upon a topic or issue that is usually presented to them as a series of questions, although sometimes as a film, a collection of advertisements, cards to sort, a game to play, or a vignette to discuss. The distinctive feature of the focus group method is its generation of interactive data. 